Ann. Génét. Sél. anim., 1976, 8 (I), 37-40.

NOTE

\title{
NOUVELLES OBSERVATIONS SUR UNE TRANSLOCATION ROBERTSONIENNE EN RACE BOVINE ROMAGNOLE
}

\author{
G. SUCCI, Annamaria DE GIOVANNJ* et L. MOLTENI \\ Istituto di Zootecnia Generale, \\ * Istituto di Anatomia e Fisiologia degli Animali domestici, \\ Facoltà di Agraria dell'Università degli Studi di Milano, \\ Via Celoria, 2, \\ 20133 . Milan (Italie)
}

\section{RÉSUMÉ}

Dans le principal centre italien d'insémination artificielle (Cesena, prov. Forli) de la race Romagnole, une étude cytogénétique préliminaire sur les neuf taureaux utilisés a montré chez deux d'entre eux un nombre de base des chromosomes réduit à 59, avec la présence d'un chromosome submétacentrique conséquence d'une translocation $1 / 29$. Ces deux taureaux porteurs de l'anomalie, sans aucun lien de parenté, étaient phénotypiquement normaux et avaient une fertilité normale.

Parmi les translocations robertsoniennes actuellement connues chez les bovins, celle qui implique le plus grand chromosome et, probablement, le plus petit (x/29) est la plus répandue.

Observée pour la première fois en Suède par RockBorn et Gusravsson (1964) elle a été retrouvée depuis dans plusieurs pays et dans une quinzaine de races (Gustavsson, 1974).

Après avoir effectué une première étude cytogénétique dans un centre italien d'insémination artificielle (De Grovannr et al., r975), nous avons orienté notre intérêt vers la race Romagnole. Cette orientation est justifiée par des travaux antérieurs qui ont mis en évidence, dans cette race, l'existence de la fusion centrique $1 / 29$.

En effet Rugiati et Fedrigo (1967, 1968) ont trouvé, sur quatre animaux Romagnoles étudiés, deux porteurs de cette anomalie, dont un à l'état homozygote.

Nous présentons, dans cette note, le premier résultat d'une étude portant sur les taureaux utilisés actuellement dans le centre d'insémination artificielle de Cesena (province de Forli), principal centre pour la race Romagnole. 


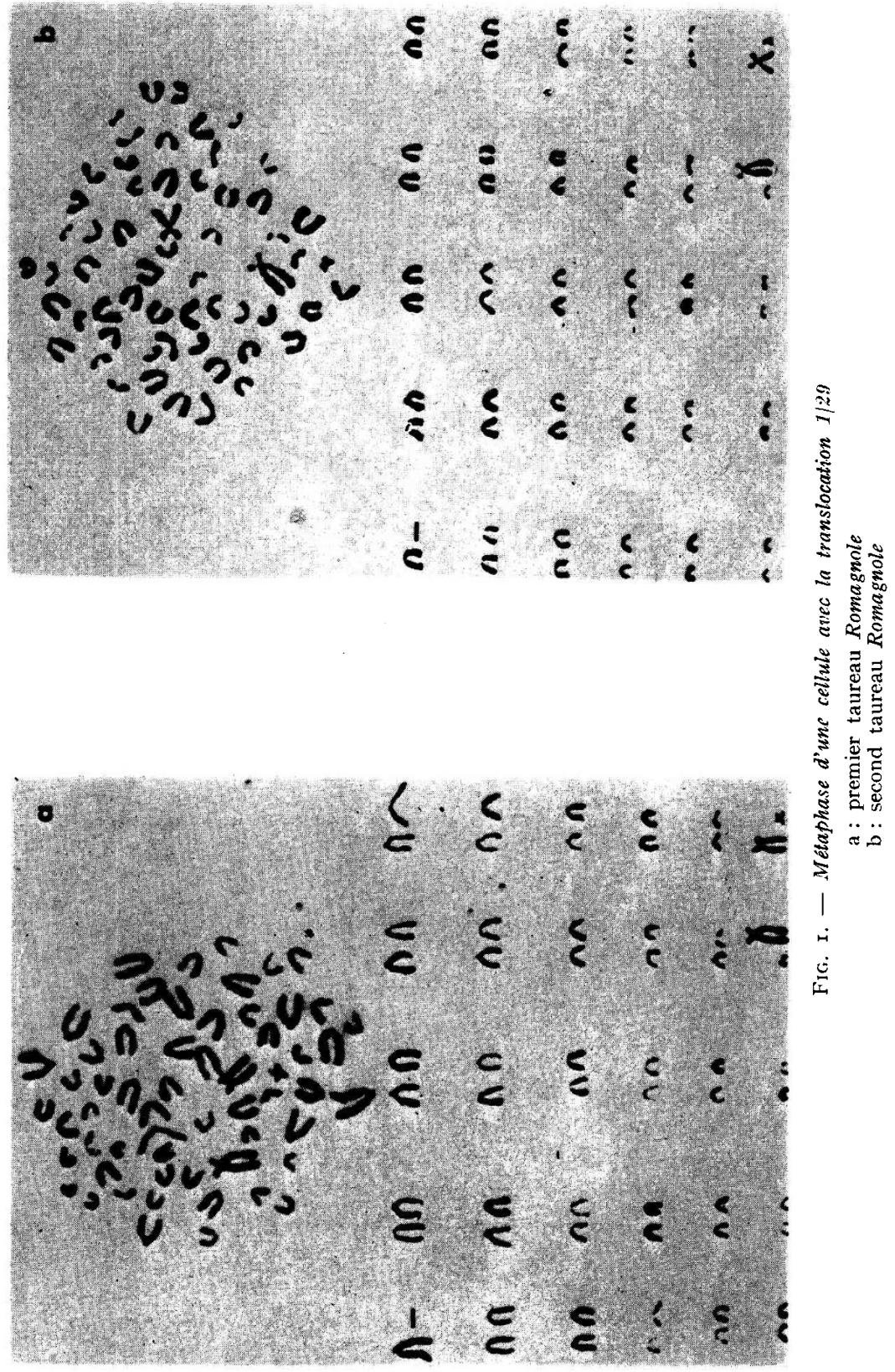


L'étude cytogénétique a été pratiquée sur des cultures de sang périphérique faites selon les méthodes de De Grouchy et al. (1964). Un grand nombre de métaphases a été étudié pour chaque animal, dont certaines ont été " caryotypées".

Sur les neuf taureaux examinés, sept présentaient un caryotype normal $60, \mathrm{XY}$.

Chez les deux autres nous avons trouvé, dans toutes les cellules étudiées, le nombre de base réduit à 59 et la présence d'un chromosome submétacentrique de type $1 / 29$ (fig. I $a, b$ ). Les deux animaux porteurs de l'anomalie, sans aucun lien de parenté, étaient phénotypiquement normaux et avaient une fertilité normale.

Les quatre animaux Romagnoles étudiés précédemment par Rugiati et Fediso (1968) présentaient tous un syndrome chondro-distrophyque, ce qui donnait à penser à une liaison éventuelle avec la fusion centrique.

Les études effectuées par la suite portant sur de nombreux animaux de différentes races, ainsi que les animaux de race Romagnole, qui font l'objet de cette note, ont montré que la fusion centrique $1 / 29$, comme la plupart des anomalies structurales, n'a aucun effet phénotypique visible. Néanmoins, elle est à l'origine de troubles de fertilité dus à l'augmentation de la mortalité embryon naire (Gustavsson, 1969, 1970).

En raison de l'intérêt scientifique et pratique de l'étude cytogénétique des bovins, nous envisageons d'entreprendre une enquête systématique sur les animaux de race Romagnole pour mieux connaître la fréquence de cette anomalie, la présence éventuelle d'anomalies nouvelles et leurs effets.

Recu four publication en janvier 1976.

\author{
SUMMARY \\ NEW OBSERVATIONS ON A ROBERTSONIAN TRANSLOCATION \\ IN THE ROMAGNOLA BREED
}

A cytogenetic study has been performed on nine bulls of Romagnola breed. The bulls belonged to the biggest Italian A.I. Center for this breed of Cesena prov. Forli.

Two bulls had only 59 chromosomes and a submetacentric chromosome was present, due to a $1 / 29$ translocation. These two bulls, with no relation to each other, were phenotypically normal and had a normal fertility. This result confirms some previous examinations on the existence of $1 / 29$ ttranslocation in Romagnola cattle but infirms the association of this chromosomal anomaly with an hereditary condrodis, strophy present in the breed.

\title{
RIASSUNTO
}

\section{NUOVE OSSERVAZIONI SU UNA TRASLOCAZIONE ROBERTSONIANA NEILA RAZZA ROMAGNOIA}

Nelo principale centro di fecondazione artificiale di Cesena (Forlí, Italia) della razza bovina Romagnola, è stato effettuato uno studio citogenetico preliminare sui nove tori utilizzati.

In due animali è stato trovato il numero base dei cromosomi ridotto a 59 e la presenza di un cromosoma submetacentrico a seguito di una traslocazione $\mathrm{r} / 29$.

I due tori portatori dell'anomalia, senza legame di parentela, erano fenotipicamente normali ed avevano una fertilità normale. 


\section{RÉFÉRENCES BIBLIOGRAPHIQUES}

1)e Grovann A. M., Popesct C. P., Succl G., 1975. Première étude cytogénétique dans un centre italien d'insémination artificielle. Ann. Génét. Sél. anim., 7 (sous presse).

Je Grocchy J., Roubin M., Passage F., r964. Microtechnique pour l'étude des chromosomes humains à partir d'une culture de leucocytes sanguins. Ann. Génét. 7, 45.

Gustavsson I., r969. Cytogenetics, distribution and phenotypic effects of a translocation in Swedish cattle. Hereditas, 63-68.

Gustavssox I., 197o. Economic importance of a translocation in Swedish cattle. Europ. Kolloq. Zytogenet. (Chromosomenpathol.). V'eterinärmed. Säugetierk., (Giessen, 12-13 oct., 108-1 14 .

(iustavssox 1., 1974. Chromosomal polynorphism. Jer Congr. Mond. Génét. appl. Ellez', Madrid, 1, Igr.

Rockвoks (i., Gustarssox I., 1964. Chromosome abmormality in three cases of lymphatic leukaemia in cattle. Nature, $203,990$.

RUgiati S., Fedrigo M., 1967. Alterazione cromosomica riscontrata in un toro acondroplasico di razza Romagnola. Ateneo Parmense, Acta Bio-Med., 38 (5).

Rugiati S., Fedrigo M., 1968. Anomalie cromosomiche riscontrate in bovini condrodistrofici della razza Romagnola. Ateneo Parmense, Acta Bio-Med., 39, 457. 